\title{
Increased perceptions of autonomy through choice over one's observation schedule fail to enhance motor skill retention
}

\author{
Laura St. Germain ${ }^{1}$, Allison Williams ${ }^{1}$, Noura Balbaa ${ }^{1}$, Andrew \\ Poskus $^{1}$, Olena Leshchyshen ${ }^{1}$, Keith R. Lohse ${ }^{2}$, and \& Michael J. \\ Carter $^{1}$ \\ ${ }^{1}$ Department of Kinesiology \\ McMaster University \\ 2 Program in Physical Therapy \\ Washington University School of Medicine in Saint Louis
}

\begin{abstract}
There has been growing research interest in the effects that motivation plays in motor learning, and specifically how autonomy, competence, and social relatedness may directly benefit the learning process. Here, we present a preregistered manipulation of autonomy-support by providing learners with choice during the practice of a speed cup-stacking task. One group was given control over when a video demonstration was provided and the viewing speed. A yoked control group received an identical demonstration schedule, but without choice (as their schedule was matched to a participant with choice). Critically, we addressed a gap in the literature by adding a yoked group who was explicitly told that they were being denied choice and that their schedule was chosen by another participant. We found no statistically significant learning differences between groups, despite finding evidence that providing choice increased perceived autonomy. Equivalence tests further showed that although the groups were not statistically equivalent, the effect size is likely too small to practically study the effects of autonomy-support through choice in most motor learning labs. These findings add to a growing body of research that questions a causal role of autonomy-support on motor learning, and the robustness of the so-called self-controlled learning advantage.
\end{abstract}

Keywords: Motor learning; Pre-registered; Self-controlled; Observation

Accepted for publication in Journal of Experimental Psychology: Human Perception \&3 Performance

A popular recommendation in recent years for creating an effective environment for motor skill learning has been to allow the learner to take control over an element of their practice that is traditionally controlled by a coach, therapist, or teacher (Sanli, Patterson, Bray, \& Lee, 2013; Ste-Marie, Carter, \& Yantha, 2019). This recommendation is based 
on the consistent finding that participants in a self-controlled (i.e., choice) group perform with higher proficiency compared to participants in a yoked (i.e., control) group on delayed retention and/or transfer tests. Participants in the yoked group do not experience the same choice opportunity provided to those in the self-controlled group. Instead, they are linked to a self-controlled participant and experience this participant's self-selected practice schedule. This so-called self-controlled learning advantage has been shown when participants are given the opportunity to schedule task difficulty (e.g., Andrieux, Danna, \& Thon, 2012; Leiker et al., 2016), the order that multiple tasks are practiced (e.g., Wu \& Magill, 2011), the frequency of watching a modeled demonstration (e.g. Wulf, Raupach, \& Pfeiffer, 2005), and when to receive augmented feedback (e.g., Janelle, Barba, Frehlich, Tennant, \& Cauraugh, 1997; Patterson \& Carter, 2010).

Over the years, this manipulation has been described using a variety of names (e.g., self- and/or learner-controlled; -regulated; -directed; -selected), but more recently some researchers have adopted the term autonomy-support. Within their "OPTIMAL" theory of motor learning, Wulf and Lewthwaite (2016) argued that providing learners with opportunities for choice creates an autonomy-supportive practice environment, which facilitates motor performance and learning. Specifically, the authors predict that autonomysupport facilitates performance by enhancing expectancies (Prediction 3, p. 1404), that enhanced expectancies and autonomy support contribute to efficient goal-action coupling by readying the motor system for task execution (Prediction 2, p. 1404), and that enhanced expectancies and autonomy support facilitate motor learning by making dopamine available for memory consolidation and neural pathway development (Prediction 7, p. 1404). In other words, these psychological benefits of increased perceptions of autonomy and competence, and the resulting increases in performance and learning are a by-product of having choice itself. Overall, Wulf and Lewthwaite's (2016) "OPTIMAL" theory of motor learning provides a motivational explanation for the learning advantages of self-controlled practice conditions over yoked practice conditions. ${ }^{1}$

\footnotetext{
${ }^{1}$ It should be noted that other researchers have instead presented an information-processing explanation for the self-controlled learning advantages (see Ste-Marie et al., 2019 for a recent discussion of the motivational and information-processing explanations). We acknowledge this view here; however, unlike previous experiments (e.g., Barros, Yantha, Carter, Hussien, \& Ste-Marie, 2019; Carter, Carlsen, \& Ste-Marie, 2014; Carter \& Ste-Marie, 2017b, 2017a; Couvillion, Bass, \& Fairbrother, 2020; Woodard \& Fairbrother, 2020) the current experiment was not designed to test between explanations. We focused on the motivational explanation as this view has garnered more attention due the "OPTIMAL" theory of motor learning.
}

Laura St. Germain (D) https://orcid.org/0000-0002-5513-4183

Keith R. Lohse (D) https://orcid.org/0000-0002-7643-3887

Michael J. Carter (iD https://orcid.org/0000-0002-0675-4271

Preregistration: https://aspredicted.org/ze8cj.pdf

Data, code, and materials: https://github.com/cartermaclab/expt_explicit-yoked

PsyArXiv preprint: https://doi.org/10.31234/osf.io/s34fg

Correspondence concerning this article should be addressed to Michael J. Carter, 1280 Main Street West, Ivor Wynne Centre Room 203, McMaster University, Hamilton ON Canada, L8S 4K1. E-mail: michaelcarter@mcmaster.ca 
Despite its prominent role as a robust and generalizable learning variable in the "OPTIMAL" theory (p. 1393), there is considerable ambiguity surrounding whether the provision of choice is in fact an autonomy-supportive manipulation. First, the notion that practicing in a self-controlled group is actually more autonomy-supportive than a yoked group has primarily been assumed (e.g., Abdollahipour, Palomo Nieto, Psotta, \& Wulf, 2017; Chua, Wulf, \& Lewthwaite, 2018; Lewthwaite, Chiviacowsky, Drews, \& Wulf, 2015; Wulf, Lewthwaite, Cardozo, \& Chiviacowsky, 2018) rather than supported empirically. Second, when researchers have included measures related to perceptions of autonomy the data is mixed. For example, Ste-Marie, Vertes, Law, and Rymal (2013) did not find the expected effect of higher perceptions of autonomy during practice in a self-controlled group as compared to a yoked group. Similar outcomes have been reported by others (e.g., Barros et al., 2019; Carter \& Ste-Marie, 2017b; McKay \& Ste-Marie, 2020a). In contrast, McKay and Ste-Marie (2020b) recently found that practicing in a self-controlled group was perceived as more autonomy-supportive than practicing the same task in a yoked group. However, despite their higher perceived autonomy scores the self-controlled group did not have significantly better motor performance and learning as predicted in the "OPTIMAL" theory. Although the majority of experiments that included a measure related to perceived autonomy reported no group differences, the absence of evidence is not evidence of absence (Altman \& Bland, 1995). Thus, self-controlled practice conditions could be an autonomy-supportive manipulation but such an effect may actually be quite small and require much larger sample sizes to detect than those used in previous experiments (e.g., Barros et al., 2019; Ste-Marie et al., 2013) and in motor learning experiments in general (see Lohse, Buchanan, \& Miller, 2016 for a discussion). This argument of underpowered experimental designs is supported by the results of McKay and Ste-Marie (2020b) as these authors had one of the largest sample sizes to date in the self-controlled literature.

There are at least two other methodological issues that warrant consideration. In self-controlled motor learning experiments participants in the self-controlled group are usually given choice over a single component (e.g., feedback or when to watch a modeled demonstration) of their practice and participants in the yoked group are not given choice over this component. However, within the context of practice itself there are other opportunities for choice that participants may explore, independent of their assigned group. Past selfcontrolled learning experiments have used a variety of motor tasks, including but not limited to basketball free throws (e.g., Aiken, Fairbrother, \& Post, 2012) and bean-bag tossing (e.g., Grand et al., 2015). Although participants in a yoked group may not have choice over their feedback schedule (or some other practice variable) while learning such tasks, this does not preclude them from opportunities for choice - and thus autonomy-support - when choosing to try different throwing techniques, speeds, and/or release points. Thus, labeling yoked groups as being devoid of choice opportunities may be a misnomer. The other issue relates to the instructions that are provided to participants in a yoked group. In the context of feedback ${ }^{2}$, these participants are typically informed that during practice they may or may not receive feedback after a given trial (e.g., Chiviacowsky \& Wulf, 2002; Patterson \& Carter, 2010). This means that participants in yoked groups are not even aware that they

\footnotetext{
${ }^{2}$ While feedback is used in this example, this issue surrounding instructions is also relevant to other practice variables commonly used in self-controlled learning experiments.
} 
have been denied an opportunity for choice, or that their feedback schedule was created by another participant who had choice over when feedback was or was not provided. These two methodological issues, either in isolation or simultaneously, may have contributed to the consistent finding in past research with self-controlled and yoked participants self-reporting numerically similar perceptions of autonomy when asked about opportunities for choice with respect to the motor task (e.g., Ste-Marie et al., 2013) or about their practice environment in general (e.g., Barros et al., 2019; Carter \& Ste-Marie, 2017b; McKay \& Ste-Marie, 2020a).

Here, we addressed the methodological limitation of past self-controlled research where participants in the yoked group are unaware that a feature of their practice environment was created by another participant in the experiment. To this end, participants learned a speed cup-stacking task in either a self-controlled group, a traditional yoked group, or a novel explicitly aware yoked group. The self-controlled group has choice over the frequency of watching a video demonstration and the video playback speed (real-time or slow motion). Participants in the traditional and explicit yoked groups were matched to a participant in the self-controlled group and experienced the observation schedule selected by this participant. The key difference being that participants in the explicit yoked group were told that the observation schedule they would experience during practice was selected by another participant, whereas participants in the traditional yoked group were not aware of this. Motor learning was assessed using a delayed retention test. We predicted that the self-controlled group would have significantly faster stacking times in retention than the traditional yoked group. In other words, we expected to replicate the typical self-controlled learning advantage. If the self-controlled learning advantage results from choice being autonomy-supportive as argued in the "OPTIMAL" theory (Wulf \& Lewthwaite, 2016), then participants in the self-controlled group should also self-report significantly higher scores for perceptions of autonomy. Also based on the view that autonomy-support is the mechanism underlying the typical self-controlled learning advantage (Wulf \& Lewthwaite, 2016), we predicted that the explicit yoked group should have significantly slower stacking times in retention. Although we found significantly higher perceptions of autonomy in the self-controlled group, there were no significant group differences in stacking times during retention. Overall, these results do not support our predictions and are inconsistent with predictions 2, 3, and 7 in the "OPTIMAL" theory of motor learning (Wulf \& Lewthwaite, 2016).

\section{Methods}

We report how we determined our sample size, all data exclusions (if any), all manipulations, and all measures in the study (Simmons, Nelson, \& Simonsohn, 2012). The experimental design and analyses were preregistered using AsPredicted.org and can be viewed here: https://aspredicted.org/ze8cj.pdf.

\section{Participants}

One-hundred and fifty university students participated in the experiment. Sample size was determined by an a priori power calculation based on our smallest comparison of interest using GLIMMPSE (https://glimmpse.samplesizeshop.org/). An early estimate for the effect of self-controlled over yoked practice was a Hedges' $g=0.63$ (McKay, Carter, \& 
Ste-Marie, 2014) and while planning this experiment this effect was estimated to be $g=$ 0.52 (Z. Yantha, personal communication, October 2019). Based on this effect, a positive correlation of $r=0.6$ between retention and pre-test as the covariate, an alpha of 0.05 , and $80 \%$ power to detect a difference between the self-controlled and traditional yoked groups, the required number of participants was 31 per group. Considering the novelty of our Explicit Yoked group, we assumed a smaller effect, $g=0.4$, between it and the Traditional Yoked group. Using this effect and the same parameters as above, this resulted in our final sample of 50 participants per group.

Participants completed the experiment in either the Self-Controlled group $\left(M_{\text {age }}\right.$ $=18.0, S D=0.34 ; 32$ females $)$, the Traditional Yoked group $\left(M_{a g e}=19.5, S D=1.89\right.$; 28 females), or the Explicit Yoked group ( $M_{a g e}=19.2, S D=1.55 ; 30$ females $)$. We collected the Self-Controlled group first as their self-selected observation schedule was required for the yoking procedure for the two other groups. Once the Self-Controlled group had been collected, participants were randomly assigned to one of the two Yoked groups. All participants provided written informed consent approved by and conducted in accordance with the University's Research Ethics Board. Participants received either $\$ 15$ or a course bonus for their participation.

\section{Material}

Participants were tasked with learning the 3-6-3 speed cup stacking sequence based on the rules of the World Sport Stacking Association (https://www.thewssa.com/). The sequence consisted of an upstack phase and a downstack phase using official Speed Stacks cups (https://www.speedstacks.com/). Participants performed the task using both their hands and had to complete an upstacking and a downstacking phase. The upstacking phase began by completing the first 3 cup pyramid, followed by the 6 cup pyramid, and then the other 3 cup pyramid. The downstacking phase began by returning to and collapsing the 3 cup pyramid that was upstacked first, then the 6 cup pyramid, and finally the last 3 cup pyramid.

\section{Procedure}

Participants completed two data collection sessions separated by approximately 24 hours. Session 1 consisted of a pre-test and an acquisition phase. Session 2 consisted of a delayed retention test. At the start of each phase of the experiment, all participants received phase-specific instructions \hyperref[sec:sharing] \{(see Data, materials, and code availability section). Group specific instructions were provided prior to the acquisition phase (see Table 1). The instructions appeared on a 22-inch computer monitor (1920x1080 resolution) positioned to the right of the participant. Participants followed along as the instructions were read aloud by the researcher.

Each trial began with participants standing at a standard height table with their hands on marked positions on the table in front of them. The 12 cups were located in upside down stacks of 3-6-3 in front of the participant. Following a "Get Ready!" prompt displayed for $1 \mathrm{~s}$ on the monitor and a constant foreperiod of $1 \mathrm{~s}$, an audiovisual "Go-signal" (green square and a beep tone) was presented. Participants were instructed to start stacking as 


\section{Table 1}

Group specific components from the instructions detailing the observation schedule that would be experienced during Acquisition.

\begin{tabular}{ll}
\hline Acquisition instructions \\
\hline Self-Controlled & $\begin{array}{l}\text { Before each trial, you will be asked whether you wish to watch a modeled } \\
\text { demonstration of the task. If you choose YES, you will then be asked } \\
\text { whether you want to watch the video in real-time or in slow-motion. }\end{array}$ \\
Traditional Yoked & $\begin{array}{l}\text { Before each trial, you may or may not watch a modeled demonstration of } \\
\text { the task based on a pre-determined schedule. If you observe a model, it } \\
\text { might be presented in real-time or in slow-motion based on the } \\
\text { pre-determined schedule. }\end{array}$ \\
& $\begin{array}{l}\text { Before each trial, you may or may not watch a modeled demonstration of } \\
\text { the task based on the schedule another participant selected. If you observe a } \\
\text { model, it might be presented in real-time or in slow-motion based on what } \\
\text { that participant selected. }\end{array}$ \\
\hline
\end{tabular}

quickly as possible following the "Go-signal" as its presentation initiated the timer. Once the upstack and downstack phases were completed, participants were instructed to press the spacebar on a keyboard located in front of them to stop the timer. If an error occurred (e.g., only completed the upstack phase then stopped the timer, forgot to hit the timer, etc), the experimenter recorded the trial number for later removal.

The pre-test and delayed retention test both consisted of five no-feedback trials. The acquisition phase had 25 trials and was the only phase where the video demonstration could be watched based on group assignment. Participants in the Self-Controlled group could decide at the start of each trial if they wanted to watch the video demonstration. If they chose to watch the video, they were then asked whether they wanted to watch it in real-time or slow motion (35\% of real-time). The real-time video was $6 \mathrm{~s}$ in duration and the slow motion version was $18 \mathrm{~s}$. To ensure a constant viewing period of $18 \mathrm{~s}$, a blank screen was shown for $12 \mathrm{~s}$ following the end of the real-time video. If they chose not to watch the video, a blank screen was shown for $18 \mathrm{~s}$. Participants in the Traditional Yoked and Explicit Yoked groups received the demonstration schedule created by a participant in the Self-Controlled group with the exception that participants in the Explicit Yoked group were made aware that this schedule was created by another participant. Feedback about stacking time (s) was displayed for $2 \mathrm{~s}$ after every acquisition trial.

To test predictions based on the "OPTIMAL" theory regarding the role of motivation, enhanced expectancies, and autonomy-support, participants completed the interest/enjoyment and perceived competence subscales from the Intrinsic Motivation Inventory (McAuley, Duncan, \& Tammen, 1989) and a custom scale regarding choice used in previous self-controlled motor learning experiments (Barros et al., 2019; Carter \& Ste-Marie, 2017b). The order of questions from each scale were randomized and each question was rated using a 7-point Likert scale. Participants answered these questions after the pre-test, after trials five and 25 of acquisition, and before the delayed retention test. The values for Cronbach's 
Table 2

Cronbach's alpha for each questionnaire at each time point.

\begin{tabular}{lllll}
\hline & After pre-test & After trial 5 & After trial 25 & Before retention \\
\hline Perceived autonomy & 0.62 & 0.79 & 0.79 & 0.84 \\
Perceived competence & 0.90 & 0.92 & 0.94 & 0.92 \\
Intrinsic motivation & 0.86 & 0.90 & 0.92 & 0.92 \\
\hline
\end{tabular}

alpha for each questionnaire at each time point are reported in Table 2.

A custom LabVIEW (National Instruments Inc.) program was created that controlled the presentation of all instructions, the video demonstrations, the timing of the experimental protocol, and recorded and stored the data for later analysis.

\section{Data analysis}

Our primary outcome measure was stacking time (i.e., response time) in seconds. Trials recorded as errors $(76 / 5250=1.45 \%)$ during data collection were manually removed prior to data analysis. For each participant, pre-test and delayed retention trials were aggregated into one block of five trials and acquisition was aggregated into five blocks of five trials. Significance level was set to 0.05 for all statistical tests. Effect sizes for omnibus tests are reported using generalized eta squared $\left(\eta_{G}^{2}\right)$ or eta squared $\left(\eta^{2}\right)$. Post hoc comparisons were conducted using Holm's correction. A Cook's distance of $\geq 1$ was used to identify any influential cases and none were identified. Statistical tests are described below.

\section{Results}

\section{Pre-registered analysis}

To test whether delayed retention (Figure 1C) was differentially impacted by the experimental group experienced during acquisition, we performed a one-way ANCOVA controlling for pre-test. As can be seen, the Self-Controlled group $(M=9.99,95 \% C I=$ $[9.68,10.31])$, the Traditional Yoked group $(M=10.18,95 \% C I=[9.86,10.50])$, and the Explicit Yoked group $(M=10.12,95 \% C I=[9.81,10.44])$ all had similar stacking times in retention (means are shown as the adjusted means controlling for pre-test). The effect of Group was not significant, $F(2,146)=.335, p=.716, \eta^{2}=.002$.

\section{Non pre-registered analyses}

\section{Traditional self-controlled learning advantage}

To investigate whether a traditional self-controlled learning advantage existed - that is a comparison between the Self-Controlled and Traditional Yoked groups - we analyzed the non-adjusted retention scores using a Welch's $t$-test. The analysis revealed that the Self-Controlled group $(M=9.77,95 \% C I=[9.55,9.99])$ and the Traditional Yoked group $(M=10.20,95 \% C I=[9.92,10.49])$ were not statistically different, $t(88.10)=1.45, p=.15$, $g=.29$. 

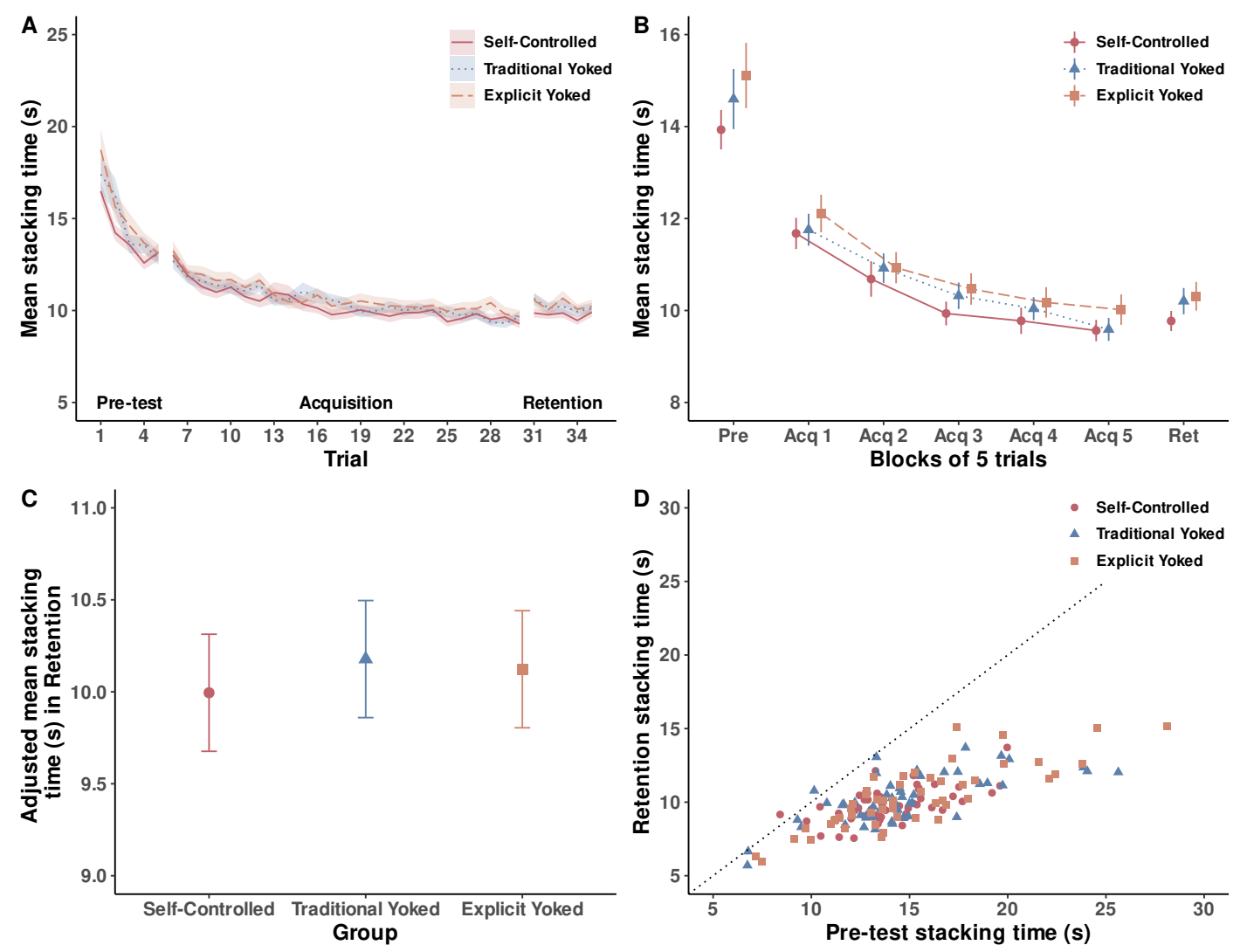

Figure 1

Physical performance data of the experiment. The Self-Controlled group is shown in red with solid lines and/or circles, the Traditional Yoked group is shown in blue with dotted and/or triangles, and the Explicit Yoked group is shown in orange with dashed and/or squares. (A). Trial-by-trial stacking time (s) data averaged across participants within each group (shaded area represents standard error). Splits between line segments denote the end of one phase and the start of the next. Pre-test (Trials 1 to 5) and Acquisition (Trials 6 to 30) occurred on Day 1 and Retention (Trials 31 to 35) occurred on Day 2, approximately 24-hours later. (B). Mean stacking time (s) for each group was computed by averaging the data from A into 7 blocks of trials. Error bars represent $95 \%$ confidence intervals. Splits between line segments denote the end of one phase and the start of the next. (C). Mean stacking time (s) adjusted for pre-test for each group. Error bars represent 95\% confidence intervals. (D). Scatterplot showing the relationship between each participant's mean stacking time (s) in pre-test and retention. 


\section{Table 3}

The mean frequencies for each Acquisition block of requesting to watch the video demonstration and of those requests, the mean frequency of choosing the regular viewing speed.

\begin{tabular}{llllll}
\hline & Block 1 & Block 2 & Block 3 & Block 4 & Block 5 \\
\hline Model viewing frequency & $32.6 \%$ & $16.9 \%$ & $12.0 \% \%$ & $12.9 \%$ & $9.3 \%$ \\
Regular speed frequency & $33.8 \%$ & $38.1 \%$ & $50.0 \%$ & $34.4 \%$ & $30.4 \%$ \\
\hline
\end{tabular}

\section{Acquisition phase}

Model frequency and the video speed request data are reported in Table 3. The mean frequency of viewing the video demonstration during acquisition was $16.7 \%$. When a video demonstration was selected, the mean frequency that the regular speed was selected was $37.3 \%$. Stacking time for each Group decreased across acquisition trials (Figure 1A) and blocks (Figure 1B). A 3 Group x 5 Block mixed ANOVA with repeated measures on Block revealed a significant Block effect, $F(3.63,533.79)=116.73, p<.001, \eta_{G}^{2}=.133$. Post hoc comparisons revealed all acquisition blocks were significantly different from each other. Both the Group effect, $F(2,147)=.738, p=.48, \eta_{G}^{2}=.008$, and Group x Block interaction, $F(7.26,533.79)=.543, p=.802, \eta_{G}^{2}=.001$, were not statistically significant.

\section{Perceived autonomy}

Self-reported perceived autonomy scores are displayed in Figure 2A where it can be seen that the Self-Controlled group reported slightly higher scores compared to the two Yoked groups at all time points following the pre-test. A 3 Group x 3 Time ANCOVA controlling for pre-test revealed a significant Group effect, $F(2,146)=8.04, p<.001, \eta_{G}^{2}=.083$. Post hoc comparisons on the adjusted means revealed that the Self-Controlled group $(M=5.61$, $95 \% C I=[5.42,5.80])$ had significantly higher perceptions of autonomy compared to both the Traditional Yoked group $(M=5.13,95 \% C I=[4.94,5.32])$ and the Explicit Yoked group $(M=5.13,95 \% C I=[4.94,5.32])$, which did not differ from each other (and were, in fact, identical to two decimal places). Both the Time effect, $F(1.61,234.98)=.867, p=.400$, $\eta_{G}^{2}=.001$, and Group x Time interaction, $F(3.22,234.98)=2.10, p=.096, \eta_{G}^{2}=.005$, were not significant.

\section{Intrinsic motivation}

Self-reported intrinsic motivation scores can be found in Figure 2B. At each time point, all groups reported similar scores on the interest/enjoyment subscale. A 3 Group x 3 Time ANCOVA controlling for pre-test revealed non-significant effects for Group, $F(2,146)=2.08$, $p=.129, \eta_{G}^{2}=.024$, Time, $F(1.68,245.73)=.563, p=.541, \eta_{G}^{2}=.001$, and the Group x Time interaction, $F(3.37,245.73)=.333, p=.824, \eta_{G}^{2}=.001$.

\section{Perceived competence}

The scores from the perceived competence subscale at each time point are displayed in Figure 2C. As can be seen, perceived competence scores showed a modest increase over 

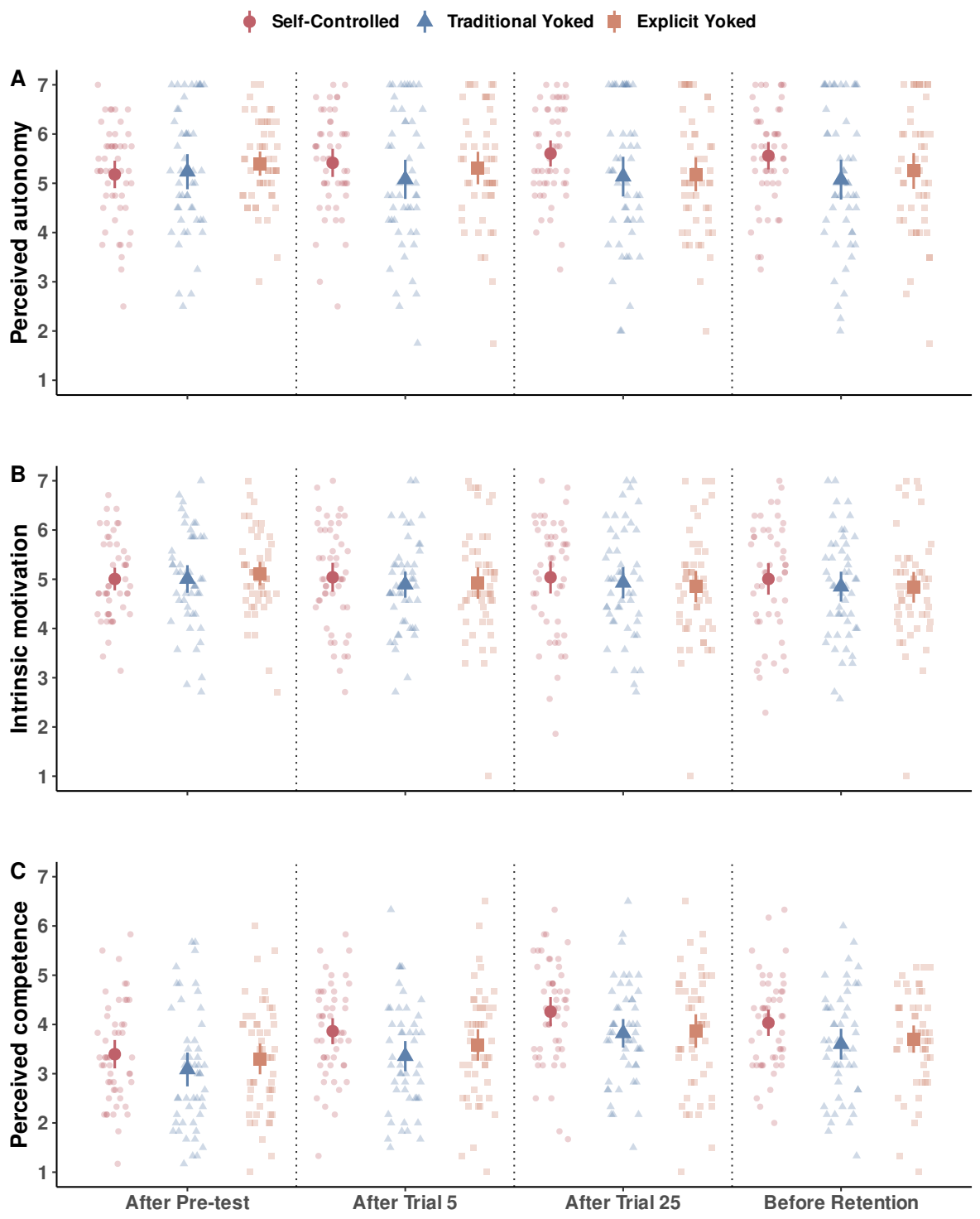

\section{Figure 2}

Questionnaire data. Groups means for perceived autonomy (A), intrinsic motivation $(\boldsymbol{B})$, and perceived competence $(\boldsymbol{C})$ after pre-test, trial 5, and trial 25 on Day 1, and before retention on Day 2. The Self-Controlled group is shown in red circles, the Traditional Yoked group is shown in blue triangles, and the Explicit Yoked group is shown in orange squares. Dots represent individual participants in each group. Scores could range from 1 (Strongly disagree) to 7 (Strongly agree). Error bars represent 95\% confidence intervals. 
time followed by a slight decrease before the delayed retention test. A 3 Group x 3 Time ANCOVA controlling for pre-test showed an effect of Time, $F(1.75,255.22)=10.60, p<.001$, $\eta_{G}^{2}=.015$. Post hoc tests on adjusted means revealed that scores were rated as significantly higher at the end of acquisition $(M=3.98,95 \% C I=[3.67,3.89])$ and before retention $(M=3.78,95 \% C I=[3.67,3.89])$ compared to after trial $5(M=3.60,95 \% C I=[3.49,3.71])$. However, perceived competence was significantly lower before retention compared to the end of acquisition. No significant effect of Group, $F(2,146)=2.65, p=.074, \eta_{G}^{2}=.028$, or Group x Time interaction, $F(3.50,255.22)=.549, p=.677, \eta_{G}^{2}=.002$, was found.

\section{Equivalence tests}

Given our pre-registered analysis resulted in a non-significant finding, we performed two equivalence tests (Self-Controlled versus Traditional Yoked and Traditional Yoked versus Explicit Yoked) on pre-test adjusted retention stacking times using the two one-sided test procedure (Lakens, Scheel, \& Isager, 2018; Schuirmann, 1987). Equivalence tests are a statistical tool that researchers can use to support the absence of a meaningful effect and avoid incorrectly concluded no effect exists based on a non-significant finding (Harms \& Lakens, 2018). To establish our smallest effect size of interest (Lakens, Pahlke, \& Wassmer, 2021) we calculated the effect size our design had 33\% power to detect (Simonsohn, 2015), which was $d_{s}=.31$. The Self-Controlled and Traditional Yoked groups were not statistically different, $t(97.99)=-0.830, p=0.409$, and not statistically equivalent, $t(97.99)=0.720$, $p=0.237$, given equivalence bounds of -.355 and .355 (on a raw scale). Similarly, the Traditional Yoked and Explicit Yoked groups were not statistically different, $t(97.99)=0.262$, $p=0.794$, and not statistically equivalent, $t(97.99)=-1.288, p=0.100$, given equivalence bounds of -.355 and .355 (on a raw scale).

\section{Discussion}

It has been argued that self-controlled practice conditions are effective for motor learning because they are autonomy-supportive in nature (Wulf \& Lewthwaite, 2016). Yet, this claim has received, at best, modest support in the motor learning literature (e.g., McKay \& Ste-Marie, 2020b; but see Barros et al., 2019; Carter \& Ste-Marie, 2017b; Ste-Marie et al., 2013 for non-support). Here, we addressed a possible methodological limitation for this lack of support. That is, in previous self-controlled motor learning research, participants in the yoked group are not made aware that they have been denied opportunities to exercise choice over a practice variable such as feedback or watching a modeled demonstration. In the present experiment we introduced a novel yoked group that was explicitly told that the observation schedule - frequency and speed of video - they would receive during practice was actually one created by another participant in the experiment. Contrary to our prediction, the Explicit Yoked group did not report significantly lower perceived autonomy scores than the Traditional Yoked group, which was only informed their observation schedule was predetermined. In line with the "OPTIMAL" theory (Wulf \& Lewthwaite, 2016), the Self-Controlled group reported significantly higher perceived autonomy-support than both the Traditional and Explicit Yoked groups. However, this boost in perceived autonomy did not lead to enhanced motor performance or learning. These findings from a preregistered experiment are difficult to reconcile with a core pillar of the "OPTIMAL" theory of motor 
learning.

\section{Self-controlled practice conditions as autonomy-supportive}

A common problem with many of the self-controlled or choice motor learning literature claiming autonomy-support as an underlying mechanism for self-controlled learning advantages is a failure to include measures that actually test this claim (e.g., Chiviacowsky, 2014; Chiviacowsky, Wulf, \& Lewthwaite, 2012; Lewthwaite et al., 2015). Instead, this link to autonomy-support is merely assumed based on the provision of choice to one group of participants versus not giving the same choice to another group of participants. As noted earlier, an issue with this assumption is that within the practice environment itself, there are myriad opportunities for participants, independent of group, to exercise choice. In throwing tasks common to motor learning, participants can explore the task workspace by attempting different throwing techniques. In more lab-based tasks that consist of multiple spatial and timing goals (e.g., waveform matching), participants can choose a single goal to focus on and master before shifting their attention to another goal. Commensurate with this idea, Ste-Marie et al. (2013) found no differences in perceived choice regarding the motor task being learned between their self-controlled and yoked groups, despite the self-controlled group showing enhanced retention. This may explain why research has consistently reported perceived autonomy scores that do not differ significantly between self-controlled and yoked groups (Barros et al., 2019; Carter \& Ste-Marie, 2017b; McKay \& Ste-Marie, 2020a).

Another possible explanation for the finding that being in a self-controlled group has not been perceived as more autonomy-supportive than being in a yoked group is that choice is typically provided over a single element of the practice variable, such as the frequency of receiving feedback (e.g., Aiken et al., 2012; Carter \& Ste-Marie, 2017a) or the level of task difficulty (Andrieux et al., 2012; Leiker et al., 2016). Control or choice over a single dimension may not be strong enough to elicit a large enough boost in perceived autonomy above and beyond that of being able to explore one's task workspace as mentioned above. Thus, to increase the saliency of the self-controlled manipulation in the present experiment, we gave the self-controlled participants control over two elements of their observation schedule: viewing frequency and video playback speed. Having control of these two dimensions resulted in higher perceptions of autonomy in our Self-Controlled group compared to the Traditional and Explicit Yoked groups. This may explain the inconsistency with past research failing to find this effect (Barros et al., 2019; Carter \& Ste-Marie, 2017b; McKay \& Ste-Marie, 2020a; Ste-Marie et al., 2013). However, McKay and Ste-Marie (2020b) recently found that choice over a single element increased perceived autonomy, but similar to our data they also did not find this led to increased learning. Thus, it is unclear whether the higher autonomy scores in the present experiment can in fact be attributed to having control over multiple elements of one's observation schedule.

In a similar vein, past research has also suggested that self-controlled learning benefits are not dependent on the amount of choice opportunities over a single element (Patterson, Carter, \& Sanli, 2011). Given these findings, we argue that the most likely explanation for the inconsistency surrounding the effect of self-controlled practice being autonomy-supportive is that this effect is quite small, and the designs of previous experiments have lacked the statistical power to reliability detect this effect. These experiments have had sample sizes 
of 20 participants or less per group, whereas there were 50 participants in each group in the present experiment and 64 per group (when collapsed across constant and variable practice schedules) in McKay and Ste-Marie (2020b). Collapsing across our two yoked groups, we estimate an effect of autonomy-support of $g=.197$ whereas the estimate from McKay and Ste-Marie (2020b) was $g=.57$. Regardless of the estimated size of the effect for autonomy-support through a self-controlled practice condition, a larger issue and challenge to the "OPTIMAL" theory is that significantly higher perceptions of autonomy did not result in superior performance in either acquisition or delayed retention (Wulf \& Lewthwaite, 2016).

\section{No learning advantage from a self-controlled observation schedule}

The delayed retention results in the present experiment not only are inconsistent with numerous past experiments reporting a self-controlled motor learning benefit (for a review see Ste-Marie et al., 2019), but also the general consensus that the self-controlled learning advantage is a robust effect (Wulf \& Lewthwaite, 2016). Of late, however, there has been an increase in the number of papers reporting a failure to detect the so-called self-controlled learning advantage (e.g., Barros et al., 2019; Grand, Daou, Lohse, \& Miller, 2017; McKay \& Ste-Marie, 2020b, 2020a). This recent lack of support may arise from the self-controlled learning advantage being a much smaller effect than originally estimated $(g=0.63$ by McKay et al. 2014). A more recent estimate of this effect from a meta-analysis using a weight-function model was $g=.11,95 \% C I=[.047, .18]$ (McKay, Yantha, Hussien, Carter, $\&$ Ste-Marie, Accepted). This estimate was further reduced to $g=.054$ after controlling for publication bias using the precision-effect estimate with standard error (PEESE) method. Thus, both models seem to suggest a trivial effect for self-controlled learning. Additional simulations included in the meta-analysis provided plausible effect size estimates ranging from $g=-.11$ to .26 (i.e., it could even favour being in the yoked group). In our experiment, the estimated effect size of self-controlled versus yoked (collapsed across our Traditional and Explicit Yoked groups) was $g=0.22$. While this estimate is larger than those from the weight-function model and PEESE method, it does fall within the range of plausible effect sizes (McKay et al., Accepted). When the lack of a self-controlled advantage in previous work and our current experiment are contextualized within the findings of this recent meta-analysis, it is not as surprising that the so-called self-controlled learning benefit was not replicated.

Despite the large sample size of the present experiment $(n$ /group $=50)$ versus the typical self-controlled motor learning experiment (median $n$ /group $=18$ based on the experiments included in the meta-analysis by McKay et al. 2021), the results of the primary analysis and the equivalence tests remain inconclusive. This, along with the effect size estimates in the recent meta-analysis, suggest enormous sample sizes are required to reliably detect an effect of a self-controlled learning advantage. Using the upper bound, $g=.26$, of the range of plausible effects, 253 participants are required to have $80 \%$ power to detect this effect in retention using an independent-samples $t$-test. The number of participants jumps to 1300 per group, if for instance the estimate from the weight-function model, $g=.11$, is accurate (McKay et al., Accepted). Given the field of motor learning suffers from a lack of adequately powered designs (Lohse et al., 2016) and that underpowered designs are more 
likely to produce false positives and overestimated effect sizes (Button et al., 2013), we are skeptical of the replicability of the previous overwhelming support for the self-controlled learning advantage.

\section{Lack of support for key predictions of the "OPTIMAL" theory of motor learning}

Within the "OPTIMAL" theory (Wulf \& Lewthwaite, 2016), it is predicted that practice conditions that promote autonomy-support and enhance expectancies contribute to a virtuous cycle that leads to superior motor performance and learning. In other words, significant group differences between self-controlled and yoked groups would be expected on measures related to these psychological constructs. As mentioned earlier, we found higher perceptions of autonomy-support in our Self-Controlled group relative to the Traditional and Explicit Yoked groups; however, this did not enhance motor learning as predicted within the virtuous cycle. Contrary to the "OPTIMAL" theory, we did not find an effect of group for perceived competence (i.e., enhanced expectancies) or intrinsic motivation. Perceived competence scores did increase across acquisition blocks, which mirrors the improved motor performance seen throughout acquisition. These results are in line with results of a path analysis that suggested self-efficacy (i.e., enhanced expectancies) and intrinsic motivation were insufficient to explain self-controlled learning benefits (Ste-Marie, Carter, Law, Vertes, \& Smith, 2016). Overall, our results are inconsistent with key predictions of the "OPTIMAL" theory regarding autonomy-support and enhanced expectancies, and further question the causal role such manipulations play in motor learning.

In sum, we found that being able to control two aspects of one's observation schedule during practice was perceived as autonomy-supportive relative to not having this same control opportunity. However, making participants in an Explicit Yoked group aware they would be denied a control opportunity during practice did not decrease perceptions of autonomy relative to participants in the Traditional Yoked group. Furthermore, we did not find evidence than an autonomy-supportive manipulation had any direct causal effect on learning and we also failed to replicate the typical self-controlled learning advantage. It is worth noting that in some situations autonomy-support in and of itself might be a desired affective outcome (Ste-Marie, Lelievre, \& Germain, 2020). In such circumstances, providing learners with choice opportunities may be a useful motivationally-protective outcome. However, such a tool should be used judiciously as it is typically motor outcomes in the form of relatively permanent performance changes that is the true goal of any motor learning intervention (Ste-Marie et al., 2020). Ultimately, our results add to a growing body of evidence that questions whether autonomy-supportive manipulations directly affect motor learning. One explanation for this lack of replicability is that the "true" effect of such manipulations are much smaller than previously estimated (McKay et al., Accepted). Given the resources required (in terms of sample size) to reliably detect these tiny effects, we encourage motor learning scientists to invest their limited resources carefully - either by adopting the use of sequential analyses (Lakens, 2014; Lakens et al., 2021; Wald, 1945) and/or multi-lab collaborations (Boland, Karczewski, \& Tatonetti, 2017) or studying practice conditions that likely have much bigger effects on motor learning. 


\section{Contributions using CrediT (https://casrai.org/credit/)}

Conceptualization (LSG, KRL, MJC); Data curation (LSG, KRL, MJC); Formal analysis (LSG, KRL, MJC); Funding acquisition (MJC); Investigation - Performed Experiment (LSG, AW, NB, AP, OL); Methodology (LSG, KRL, MJC); Project administration (LSG, MJC); Resources (MJC); Software - Task Programming (LSG, MJC); Supervision (MJC); Validation (LSG, KRL, MJC); Visualization (LSG, MJC); Writing - Original Draft Preparation (LSG, AW, NB, KRL, MJC); Writing - Review \& Editing (LSG, AW, NB, AP, OL, KRL, MJC)

\section{Data, materials, and code availability}

All material, data, and scripts to reproduce our analyses and figures can be accessed here: https://github.com/cartermaclab/expt_explicit-yoked. The preregistration can be accessed here: https://aspredicted.org/ze8cj.pdf.

\section{$R$ packages used in this project}

$\mathrm{R}$ (Version 4.0.4; R Core Team, 2021) and the R-packages cowplot (Version 1.1.1; Wilke, 2020), ggplot2 (Version 3.3.5; Wickham, 2016), jmv (Version 2.0; Selker, Love, \& Dropmann, 2020), kableExtra (Version 1.3.4; Zhu, 2021), papaja (Version 0.1.0.9997; Aust \& Barth, 2020), pwr (Version 1.3.0; Champely, 2020), tidyverse (Version 1.3.1; Wickham et al., 2019), and TOSTER (Version 0.4.0; Lakens, 2017).

\section{Conflict of interest}

The authors declare no competing interests.

\section{Funding}

This work was supported by the Natural Sciences and Engineering Research Council (NSERC) of Canada (RGPIN-2018-05589; MJC). LSG was supported by an NSERC Postgraduate Scholarship. AW and AP were supported by NSERC Undergraduate Student Research Awards. 


\section{References}

Abdollahipour, R., Palomo Nieto, M., Psotta, R., \& Wulf, G. (2017). External focus of attention and autonomy support have additive benefits for motor performance in children. Psychology of Sport and Exercise, 32, 17-24. https://doi.org/10.1016/j.psychsport.2017. 05.004

Aiken, C. A., Fairbrother, J. T., \& Post, P. G. (2012). The effects of self-controlled video feedback on the learning of the basketball set shot. Frontiers in Psychology, 3. https://doi.org/10.3389/fpsyg.2012.00338

Altman, D. G., \& Bland, J. M. (1995). Statistics notes: Absence of evidence is not evidence of absence. BMJ, 311(7003), 485. https://doi.org/10.1136/bmj.311.7003.485

Andrieux, M., Danna, J., \& Thon, B. (2012). Self-control of task difficulty during training enhances motor learning of a complex coincidence-anticipation task. Research Quarterly for Exercise and Sport, 83(1), 27-35. https://doi.org/10.1080/02701367.2012.10599822

Aust, F., \& Barth, M. (2020). papaja: Create APA manuscripts with $R$ Markdown. Retrieved from https://github.com/crsh/papaja

Barros, J. A. C., Yantha, Z. D., Carter, M. J., Hussien, J., \& Ste-Marie, D. M. (2019). Examining the impact of error estimation on the effects of self-controlled feedback. Human Movement Science, 63, 182-198. https://doi.org/10.1016/j.humov.2018.12.002

Boland, M. R., Karczewski, K. J., \& Tatonetti, N. P. (2017). Ten simple rules to enable multi-site collaborations through data sharing. PLOS Computational Biology, 13, 1-12. https://doi.org/10.1371/journal.pcbi.1005278

Button, K. S., Ioannidis, J. P. A., Mokrysz, C., Nosek, B. A., Flint, J., Robinson, E. S. J., \& Munafò, M. R. (2013). Power failure: why small sample size undermines the reliability of neuroscience. Nature Reviews Neuroscience, 14(5), 365-376. https: //doi.org/10.1038/nrn3475

Carter, M. J., Carlsen, A. N., \& Ste-Marie, D. M. (2014). Self-controlled feedback is effective if it is based on the learner's performance: A replication and extension of chiviacowsky and wulf (2005). Frontiers in Psychology, 5. https://doi.org/10.3389/fpsyg.2014.01325

Carter, M. J., \& Ste-Marie, D. M. (2017a). An interpolated activity during the knowledgeof-results delay interval eliminates the learning advantages of self-controlled feedback schedules. Psychological Research, 81(2), 399-406. https://doi.org/10.1007/s00426-016$0757-2$

Carter, M. J., \& Ste-Marie, D. M. (2017b). Not all choices are created equal: Task-relevant choices enhance motor learning compared to task-irrelevant choices. Psychonomic Bulletin E3 Review, 24(6), 1879-1888. https://doi.org/10.3758/s13423-017-1250-7

Champely, S. (2020). Pwr: Basic functions for power analysis. Retrieved from https: //CRAN.R-project.org/package=pwr

Chiviacowsky, S. (2014). Self-controlled practice: Autonomy protects perceptions of competence and enhances motor learning. Psychology of Sport and Exercise, 15(5), 505-510. https://doi.org/10.1016/j.psychsport.2014.05.003

Chiviacowsky, S., \& Wulf, G. (2002). Self-controlled feedback: Does it enhance learning because performers get feedback when they need it? Research Quarterly for Exercise and Sport, 73(4), 408-415. https://doi.org/10.1080/02701367.2002.10609040

Chiviacowsky, S., Wulf, G., \& Lewthwaite, R. (2012). Self-controlled learning: The importance of protecting perceptions of competence. Frontiers in Psychology, 3. 
https://doi.org/10.3389/fpsyg.2012.00458

Chua, L.-K., Wulf, G., \& Lewthwaite, R. (2018). Onward and upward: Optimizing motor performance. Human Movement Science, 60, 107-114. https://doi.org/10.1016/j.humov. 2018.05.006

Couvillion, K. F., Bass, A. D., \& Fairbrother, J. T. (2020). Increased cognitive load during acquisition of a continuous task eliminates the learning effects of self-controlled knowledge of results. Journal of Sports Sciences, 38(1), 94-99. https://doi.org/10.1080/02640414.2 019.1682901

Grand, K. F., Bruzi, A. T., Dyke, F. B., Godwin, M. M., Leiker, A. M., Thompson, A. G., ... Miller, M. W. (2015). Why self-controlled feedback enhances motor learning: Answers from electroencephalography and indices of motivation. Human Movement Science, 43, 23-32. https://doi.org/10.1016/j.humov.2015.06.013

Grand, K. F., Daou, M., Lohse, K. R., \& Miller, M. W. (2017). Investigating the Mechanisms Underlying the Effects of an Incidental Choice on Motor Learning. Journal of Motor Learning and Development, 5(2), 207-226. https://doi.org/10.1123/jmld.2016-0041

Harms, C., \& Lakens, D. (2018). Making 'null effects' informative: Statistical techniques and inferential frameworks. Journal of Clinical and Translational Research. https: //doi.org/10.18053/jctres.03.2017S2.007

Janelle, C. M., Barba, D. A., Frehlich, S. G., Tennant, L. K., \& Cauraugh, J. H. (1997). Maximizing performance feedback effectiveness through videotape replay and a selfcontrolled learning environment. Research Quarterly for Exercise and Sport, 68(4), 269-279. https://doi.org/10.1080/02701367.1997.10608008

Lakens, D. (2014). Performing high-powered studies efficiently with sequential analyses. European Journal of Social Psychology, 44(7), 701-710. https://doi.org/10.1002/ejsp.2 023

Lakens, D. (2017). Equivalence tests: A practical primer for t-tests, correlations, and meta-analyses. Social Psychological and Personality Science, 1, 1-8. https://doi.org/10.1 $177 / 1948550617697177$

Lakens, D., Pahlke, F., \& Wassmer, G. (2021). Group sequential designs: A tutorial. Psyarxiv. https://doi.org/10.31234/osf.io/x4azm

Lakens, D., Scheel, A. M., \& Isager, P. M. (2018). Equivalence Testing for Psychological Research: A Tutorial. Advances in Methods and Practices in Psychological Science, 1(2), 259-269. https://doi.org/10.1177/2515245918770963

Leiker, A. M., Bruzi, A. T., Miller, M. W., Nelson, M., Wegman, R., \& Lohse, K. R. (2016). The effects of autonomous difficulty selection on engagement, motivation, and learning in a motion-controlled video game task. Human Movement Science, 49, 326-335. https://doi.org/10.1016/j.humov.2016.08.005

Lewthwaite, R., Chiviacowsky, S., Drews, R., \& Wulf, G. (2015). Choose to move: The motivational impact of autonomy support on motor learning. Psychonomic Bulletin 6 Review, 22(5), 1383-1388. https://doi.org/10.3758/s13423-015-0814-7

Lohse, K., Buchanan, T., \& Miller, M. (2016). Underpowered and Overworked: Problems With Data Analysis in Motor Learning Studies. Journal of Motor Learning and Development, 4(1), 37-58. https://doi.org/10.1123/jmld.2015-0010

McAuley, E., Duncan, T., \& Tammen, V. V. (1989). Psychometric properties of the intrinsic motivation inventory in a competitive sport setting: A confirmatory factor analysis. 
Research Quarterly for Exercise and Sport, 60(1), 48-58. https://doi.org/10.1080/0270 1367.1989.10607413

McKay, B., Carter, M. J., \& Ste-Marie, D. M. (2014). Self-controlled learning: A metaanalysis. Journal of sport $\&$ exercise psychology, 36, S43.

McKay, B., \& Ste-Marie, D. M. (2020a). Autonomy support and reduced feedback frequency have trivial effects on learning and performance of a golf putting task. Human Movement Science, 71 (1026124). https://doi.org/10.1016/j.humov.2020.102612

McKay, B., \& Ste-Marie, D. M. (2020b). Autonomy support via instructionally irrelevant choice not beneficial for motor performance or learning. Research Quarterly for Exercise and Sport, O(0), 1-13. https://doi.org/10.1080/02701367.2020.1795056

McKay, B., Yantha, Z. D., Hussien, J., Carter, M. J., \& Ste-Marie, D. M. (Accepted). Metaanalytic findings in the self-controlled motor learning literature: Underpowered, biased, and lacking evidential value. Meta-Psychology. https://doi.org/10.31234/osf.io/8d3nb

Patterson, J. T., \& Carter, M. (2010). Learner regulated knowledge of results during the acquisition of multiple timing goals. Human Movement Science, 29(2), 214-227. https://doi.org/10.1016/j.humov.2009.12.003

Patterson, J. T., Carter, M., \& Sanli, E. (2011). Decreasing the proportion of self-control trials during the acquisition period does not compromise the learning advantages in a self-controlled context. Research Quarterly for Exercise and Sport, 82(4), 624-633. https://doi.org/10.1080/02701367.2011.10599799

R Core Team. (2021). R: A language and environment for statistical computing. Vienna, Austria: R Foundation for Statistical Computing. Retrieved from https://www.Rproject.org/

Sanli, E. A., Patterson, J. T., Bray, S. R., \& Lee, T. D. (2013). Understanding self-controlled motor learning protocols through the self-determination theory. Frontiers in Psychology, 3. https://doi.org/10.3389/fpsyg.2012.00611

Schuirmann, D. J. (1987). A comparison of the Two One-Sided Tests Procedure and the Power Approach for assessing the equivalence of average bioavailability. Journal of Pharmacokinetics and Biopharmaceutics, 15(6), 657-680. https://doi.org/10.1007/BF01 068419

Selker, R., Love, J., \& Dropmann, D. (2020). Jmv: The 'jamovi' analyses. Retrieved from https://CRAN.R-project.org/package $=$ jmv

Simmons, J. P., Nelson, L. D., \& Simonsohn, U. (2012). A 21 Word Solution. Rochester, NY. https://doi.org/10.2139/ssrn.2160588

Simonsohn, U. (2015). Small Telescopes: Detectability and the Evaluation of Replication Results. Psychological Science, 26(5), 559-569. https://doi.org/10.1177/09567976145673 41

Ste-Marie, D. M., Carter, M. J., Law, B., Vertes, K., \& Smith, V. (2016). Self-controlled learning benefits: Exploring contributions of self-efficacy and intrinsic motivation via path analysis. Journal of Sports Sciences, 34(17), 1650-1656. https://doi.org/10.1080/ 02640414.2015.1130236

Ste-Marie, D. M., Carter, M. J., \& Yantha, Z. D. (2019). Self-controlled learning: Current findings, theoretical perspectives, and future directions. In Skill Acquisition in Sport: Research, Theory, and Practice (3rd ed.). Routledge.

Ste-Marie, D. M., Lelievre, N., \& Germain, L. S. (2020). Revisiting the applied model for 
the use of observation: A review of articles spanning 2011-2018. Research Quarterly for Exercise and Sport, 91(4), 594-617. https://doi.org/10.1080/02701367.2019.1693489

Ste-Marie, D. M., Vertes, K. A., Law, B., \& Rymal, A. M. (2013). Learner-controlled self-observation is advantageous for motor skill acquisition. Frontiers in Psychology, 3. https://doi.org/10.3389/fpsyg.2012.00556

Wald, A. (1945). Sequential tests of statistical hypotheses. The Annals of Mathematical Statistics, 16(2), 117-186. https://doi.org/10.1214/aoms/1177731118

Wickham, H. (2016). ggplot2: Elegant graphics for data analysis. Springer-Verlag New York. Retrieved from https://ggplot2.tidyverse.org

Wickham, H., Averick, M., Bryan, J., Chang, W., McGowan, L. D., François, R., ... Yutani, H. (2019). Welcome to the tidyverse. Journal of Open Source Software, 4(43), 1686. https://doi.org/10.21105/joss.01686

Wilke, C. O. (2020). Cowplot: Streamlined plot theme and plot annotations for 'ggplot2'. Retrieved from https://CRAN.R-project.org/package=cowplot

Woodard, K. F., \& Fairbrother, J. T. (2020). Cognitive loading during and after continuous task execution alters the effects of self-controlled knowledge of results. Frontiers in Psychology, 11. https://doi.org/10.3389/fpsyg.2020.01046

Wu, W. F. W., \& Magill, R. A. (2011). Allowing learners to choose. Research Quarterly for Exercise and Sport, 82(3), 449-457. https://doi.org/10.1080/02701367.2011.10599777

Wulf, G., \& Lewthwaite, R. (2016). Optimizing performance through intrinsic motivation and attention for learning: The OPTIMAL theory of motor learning. Psychonomic Bulletin \& Review, 23(5), 1382-1414. https://doi.org/10.3758/s13423-015-0999-9

Wulf, G., Lewthwaite, R., Cardozo, P., \& Chiviacowsky, S. (2018). Triple play: Additive contributions of enhanced expectancies, autonomy support, and external attentional focus to motor learning. Quarterly Journal of Experimental Psychology, 71(4), 824-831. https://doi.org/10.1080/17470218.2016.1276204

Wulf, G., Raupach, M., \& Pfeiffer, F. (2005). Self-controlled observational practice enhances learning. Research Quarterly for Exercise and Sport, 76(1), 107-111. https://doi.org/10 $.1080 / 02701367.2005 .10599266$

Zhu, H. (2021). kableExtra: Construct complex table with 'kable' and pipe syntax. Retrieved from https://CRAN.R-project.org/package=kableExtra 